\title{
Combustion of a frozen bi-dispersed aluminum-water suspension
}

\author{
Vasiliy Poryazov, and Aleksey Krainov \\ Tomsk State University, 634050 Tomsk, Russia
}

\begin{abstract}
This paper presents a combustion model of nano- and microsized aluminum mixture frozen in water. The model takes into account combustion of aluminum particles in water vapor, the motion of combustion products, the temperature and velocity differences between particles and gas. The obtained results of the combustion rate depending on pressure and mass ratio between dispersed $\mathrm{Al}$ powders are in good agreement with the experimental data described in scientific literature.
\end{abstract}

\section{Introduction}

Mixtures of aluminum and water have been studied for many years because of the high heat release and gasphase production of molecular hydrogen associated with this reaction. The combustion of aluminum with ice is studied using various mixtures of nano- and micrometersized aluminum particles as a means to generate high-temperature hydrogen at fast rates for propulsion and power applications [1]. Bimodal distributions are of interest in order to change the burning rate.

The effects of the bimodal distributions and equivalence ratio on ignition, combustion rates, and combustion efficiency are investigated in strand experiments at constant pressure and in small lab-scale [1]. The importance of mathematical modeling in solving this class of problems is obvious. The results to be obtained will be useful for the development of fundamental knowledge in the field of physics of combustion and explosion and can be used in the development and design of new types of solid propellants for rocket propulsion systems to calculate basic internal ballistic characteristics of the propulsion systems and reduce the amount of expensive experiments.

\section{Construction of the Mathematical Model}

The combustion model of nano- and microsized aluminum mixture frozen in water is constructed on the basis of the models from [2, 3]. To formulate the mathematical model the following assumptions are used: the linear burning rate of the frozen nanosized aluminum suspension in water is determined by the rate of water evaporation from the surface. The phenomena that occur in the heating front ahead of the water evaporation surface during the combustion of such a frozen suspension are ice heating to the melting point, ice melting, heating of the water layer to the boiling point, and water evaporation on the liquid surface. Together with water vapor, aluminum particles are also entrained into the gas phase, where they react with water vapor, forming gaseous hydrogen and aluminum oxide. To simulate the combustion of a frozen suspension of the nanosized aluminum powder in water we use the model of volatile combustion by A.F. Belyaev [4].

The aluminum particles in the frozen suspension are shaped as spheres and they are uniformly distributed in the ice volume. These particles react with water vapor. The reaction products are the aluminum oxide remaining on the particle surface and hydrogen:

$\left(2 \mathrm{Al}+3 \mathrm{H}_{2} \mathrm{O}=\mathrm{Al}_{2} \mathrm{O}_{3}+3 \mathrm{H}_{2}\right)$. The ignition of an aluminum particle occurs when it reaches a certain temperature. The burning rate of aluminum particles is described on the basis of the experimental data [5]. The heat transfer between the particles and the gas proceeds in accordance with the Newton law. The particle motion is driven by the friction force from the gas. Because of the small volume fraction of particles in the gas, their motion does not affect the gas motion. There is no agglomeration of particles on the condensed phase surface, and the particles do not interact with each other in the gas phase. Combustion occurs under isobaric conditions, and the pressure is independent of the distance from the burning surface.

The mathematical model of combustion of the frozen nanosized aluminum suspension in water, which is written in a coordinate system fitted to the burning surface, has the following form:

$$
\begin{gathered}
c_{2} \rho_{2}\left(\frac{\partial T_{2}}{\partial t}+u \frac{\partial T_{2}}{\partial x}\right)=\lambda_{2} \frac{\partial^{2} T_{2}}{\partial x^{2}}+ \\
+\sum_{i} 4 \pi \alpha_{i} r_{k, i}^{2} n_{i}\left(T_{3, i}-T_{2}\right) \\
\frac{\partial \rho_{2}}{\partial t}+\frac{\partial\left(\rho_{2} u\right)}{\partial x}=-\sum_{i} G_{i},
\end{gathered}
$$

\footnotetext{
Corresponding author: poryazov@,ftf.tsu.ru
} 


$$
\begin{gathered}
\frac{\partial \rho_{H_{2}}}{\partial t}+\frac{\partial\left(\rho_{H_{2}} u\right)}{\partial x}=\sum_{i} G_{i} \frac{\mu_{O_{2}}}{\mu_{A l}}, \\
c_{3, i} \rho_{3, i}\left(\frac{\partial T_{3, i}}{\partial t}+w_{i} \frac{\partial T_{3, i}}{\partial x}\right)= \\
-4 \pi \alpha_{i} r_{k, i}^{2} n_{i}\left(T_{3_{i}}-T_{2}\right)+G_{i} Q_{A l} \frac{2 \mu_{A l}}{3 \mu_{O}}, \\
\frac{\partial \rho_{3, i}}{\partial t}+\frac{\partial\left(\rho_{3, i} w_{i}\right)}{\partial x}=G_{i}, \\
\frac{\partial w_{i}}{\partial t}+w_{i} \frac{\partial w_{i}}{\partial x}=-\tau_{F t r i}, \\
\frac{\partial n_{i}}{\partial t}+\frac{\partial\left(n_{i} w_{i}\right)}{\partial x}=0, \\
P=\rho_{2}\left(\frac{m_{H_{2} O} O}{\mu_{H_{2} O}}+\frac{m_{H_{2}}}{\mu_{H_{2}}}\right) R T_{2}=\text { const }, \\
m_{H_{2} O}=\frac{\rho_{2}-\rho_{H_{2}}}{\rho_{2}}, m_{H_{2}}=\frac{\rho_{H_{2}}}{\rho_{2}} .
\end{gathered}
$$

According to [6], the heat capacity of an aluminum particle during the heating and melting process is described by dependence:

$$
c_{3, i}= \begin{cases}c_{3, \text { solid }}, & T_{3, i}<T_{n n}-\Delta T ; \\ \frac{L_{A l}}{2 \Delta T}, & T_{n n},-\Delta T<T_{3, i}<T_{n \imath}+\Delta T ; \\ c_{3, \text { liquid }}, & T_{3, i}>T_{n n}+\Delta T .\end{cases}
$$

The coordinate $x=0$ is corresponding to burningsurface area. The linear burning rate is determined by the rate of water evaporation from the surface.

The water mass flux conservation law is satisfied on the evaporation surface:

$$
\left(1-\sum_{i} M_{A l, i}\right) \rho_{1} V_{k}=\left.\rho_{2} u\right|_{x=0},
$$

The Al particle mass conservation on the evaporation surface is:

$$
M_{A l, i} \rho_{1} V_{k}=\left.\left(\rho_{3, i} w_{i}\right)\right|_{x=0} .
$$

The evaporation temperature on the surface is equal to the temperatures of the vapor and particles:

$$
\left.T_{3, i}\right|_{x=0}=\left.T_{2}\right|_{x=0}=T_{v} .
$$

The saturated vapor temperature $T_{v}$ over the surface is determined by the Clausius-Clapeyron equation:

$$
P=A \exp \left(-\frac{L}{R T}\right) \text {. }
$$

Then, for a given pressure $P$ above the burning surface, we obtain the expression for the saturated vapor temperature:

$$
T_{v}=\frac{L}{-R \ln \left(P / P_{a}\right)+L / T_{v, a}} .
$$

The heat flux from the gas phase onto the evaporation surface is spent on heating of the condensed substance to the ice melting temperature and its melting, on heating water to the evaporation temperature and its evaporation:

$$
\begin{aligned}
\left.\lambda_{2} \frac{\partial T}{\partial x}\right|_{x=0} & =\rho_{1} V\left(\left.c_{1} T_{2}\right|_{x=0}-c_{1} T_{1,0}\right)+ \\
& +\rho_{1}\left(1-\sum_{i} M_{A l, i}\right) V(L+\Lambda)
\end{aligned} .
$$

The density of the water vapor above the condensed phase surface is determined from the equation of state for an ideal gas:

$$
\begin{gathered}
\left.\rho_{2}\right|_{x=0}=\frac{\mu_{\mathrm{H}_{2} \mathrm{O}} P}{\left.R T_{2}\right|_{x=0}}, \\
P=\text { const } .
\end{gathered}
$$

The number of particles in a unit volume is determined by the formula:

$$
\left.n_{i}\right|_{x=0}=\frac{\left.\rho_{3, i}\right|_{x=0}}{(4 / 3) \pi r_{A, 0, i}^{3} \rho_{k}} .
$$

There are no gaseous reaction products near the water evaporation surface:

$$
\left.\rho_{H_{2}}\right|_{x=0}=0 .
$$

The following boundary condition is set on the boundary $x=\infty$ :

$$
\left.\frac{\partial T}{\partial x}\right|_{x=\infty}=0 .
$$

The initial conditions are:

$$
\begin{gathered}
T_{2}(x, 0)=T_{i g}, T_{3, i}(x, 0)=T_{i g}, \\
u(x, 0)=0, w_{i}(x, 0)=0, n_{i}(x, 0)=0, \\
\rho_{2}(x, 0)=p_{n} \mu / R T_{i g}, \rho_{3, i}(x, 0)=0 .
\end{gathered}
$$

The interaction force between the aluminum particles and the gas is calculated as:

$$
\begin{gathered}
\tau_{F t r, i}=\frac{F_{t r, i}}{4 / 3 \pi r_{k, i}{ }^{3} \rho_{k}}, \\
F_{t r, i}=C_{R, i} S_{m, i} \frac{\rho_{2}\left(w_{i}-u\right)\left|u-w_{i}\right|}{2} .
\end{gathered}
$$

Here the drag coefficient is described by the empirical formula:

$$
\begin{gathered}
C_{R, i}=\frac{24}{\operatorname{Re}_{i}}\left(1+0,15 \operatorname{Re}_{i}^{0,682}\right), \\
\operatorname{Re}_{i}=\frac{2 r_{k, i} \rho_{k}\left|u-w_{i}\right|}{\eta} .
\end{gathered}
$$

The heat transfer coefficient is determined as

$$
\begin{gathered}
\alpha_{i}=\frac{N u_{i} \lambda_{2}}{2 r_{k, i}}, \\
N u_{i}=2+\sqrt{N u_{l, i}{ }^{2}+N u_{t, i}{ }^{2}}, \\
N u_{l, i}=0,664 \operatorname{Re}_{i}{ }^{0,5}, N u_{t, i}=0,037 \operatorname{Re}_{i}{ }^{0,8} .
\end{gathered}
$$

The particle mass change rate during combustion is:

$$
G_{i}=-\frac{3 \mu_{O}}{2 \mu_{A l}} n \rho_{k} 4 \pi r_{A l, i}^{2} \frac{d r_{A l, i}}{d t} .
$$

The experimental research [5] shows that the burning time of an aluminum particle in water vapor is determined by its initial diameter $d_{A l, 0}$ as $\tau_{c}=b d_{A l, 0}^{2}$, where $b$ is a constant. Thereat $\frac{d r_{A l}}{d t}=-k_{A l} \frac{1}{r_{A l}}$. 


$$
G_{i}=\frac{3 \mu_{O}}{2 \mu_{A l}} n_{i} \rho_{k} 4 \pi r_{A l, i} k_{A l, i},
$$

where $k_{A l}$ is a constant.

The size of the unburnt portion of aluminum in a particle $r_{k}$ is determined by the formula

$$
r_{A l, i}=\left[\left(\frac{\mu_{A l}+3 / 2 \mu_{O}}{\mu_{A l}} r_{A l, 0, i}^{3}-\frac{\rho_{3, i}}{(4 / 3) \pi n_{i} \rho_{k, i}}\right) \frac{2 \mu_{A l}}{3 \mu_{O}}\right]^{1 / 3} \text {. }
$$

In deriving Eq. (20), it was assumed for simplicity that the aluminum oxide remaining in the particle has the shape of a spherical shell. This assumption was introduced to determine the current radius of the aluminum sphere in the course of its combustion and does not affect the burning rate of the aluminum particle because it is chosen with due allowance for experimental data.

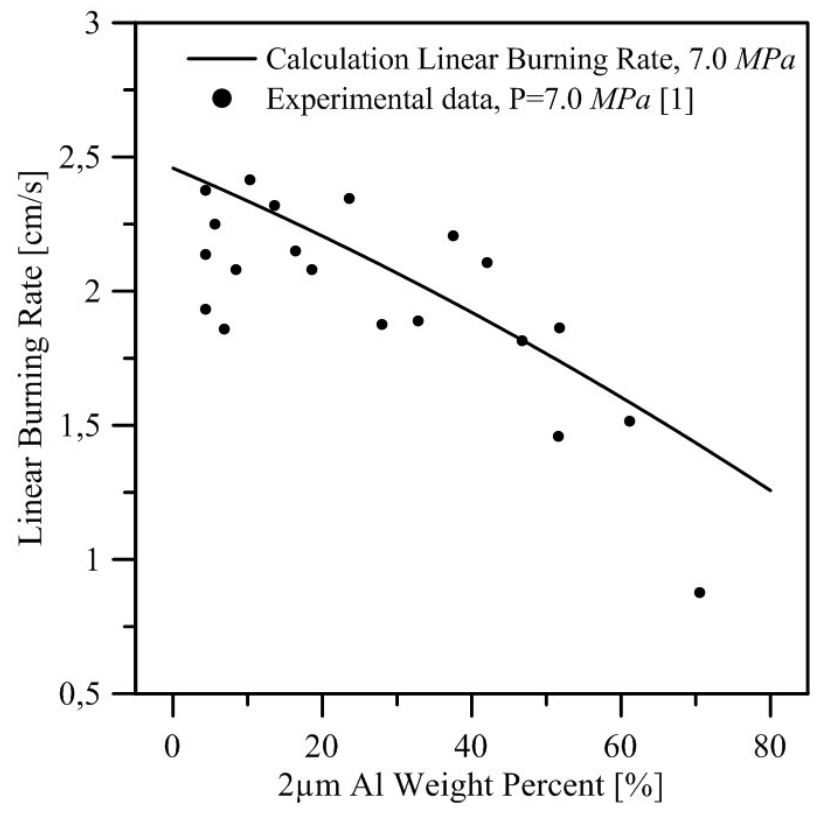

Fig.1. The burning rate of the frozen suspension depending on the micron particle mass fraction, $r_{A l, 0,1}=40 \mathrm{~nm}$, $r_{A l, 0,2}=1 \mu \mathrm{m}$

The following notation are used: $t$ is time; $x$ is coordinate; $T_{2}$ is the temperature of the gas mixture; $T_{3, i}$ is the particle temperature; $\rho_{2}$ is the gas density; $\rho_{\mathrm{H}_{2}}$ is the hydrogen partial density; $\rho_{3, i}$ is the particle reduced density (mass per unit volume); $n_{i}$ is the number of particles per unit volume; $u$ is the gas velocity; $w$ is the particle velocity; $P$ is pressure; $r_{k, i}$ is the particle radius; $m_{H_{2} O}, m_{H_{2}}$ are mass fractions of water vapor and hydrogen in total mass of gas phase; $\mu_{A l}, \mu_{O}, \mu_{H_{2}}, \mu_{H_{2} \mathrm{O}}$ are molar masses of aluminum, oxygen, hydrogen and water respectively; $c_{2}$ is the specific heat at constant pressure; $\lambda_{2}$ is the gas thermal conductivity; $\alpha_{i}$ is the heat-transfer coefficient; $G_{i}$ is the particle mass change rate during combustion; $Q_{A l}$ is the combustion heat of aluminum in water vapor; $R$ is the molar gas constant; $\tau_{F t r, i}$ is the interaction force between a particles and gas; $V_{k}$ is the linear rate of the water evaporation boundary; $M_{A l}$ is the mass fraction of Al powder in the suspension; $\rho_{1}$ is the density of frozen aluminum-water suspension; $T_{v}$ is the saturated vapor temperature; $L$ is the water boiling heat; $A$ is a constant; $c_{1}$ is the specific heat capacity of the frozen aluminum-water suspension; $\Lambda$ is the ice specific heat of melting; $T_{1,0}$ is the initial temperature of frozen suspension; $r_{A l, 0, i}$ is the initial particle radius; $\rho_{k}$ is the particle density.

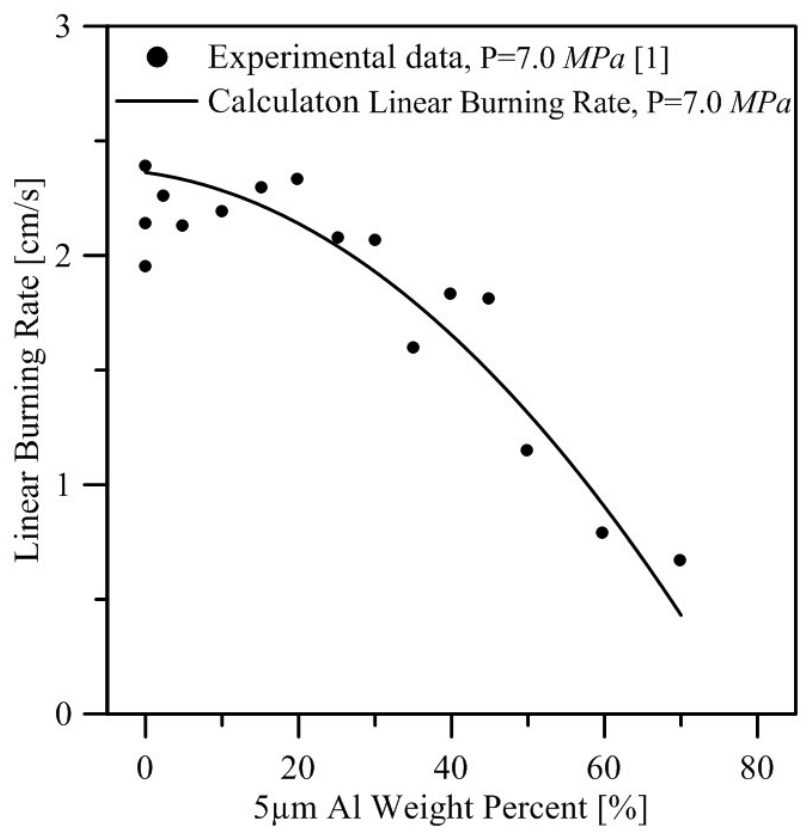

Fig.2. The burning rate of the frozen suspension depending on the micron particle mass fraction $r_{A l, 0,1}=40 \mathrm{~nm}$, $r_{A l, 0,2}=2.5 \mu \mathrm{m}$

We have conducted the numerical investigation on combustion of the frozen aluminum-water suspension with the mass fraction of the aluminum powder corresponding to a stoichiometric mixture of aluminum with water. The computations were performed for the following values of the thermophysical variables:

$\rho_{1}=1460 \mathrm{~kg} / \mathrm{m}^{3}, M_{A l}=0.499, c_{1}=c_{\text {wat }}\left(1-M_{A l}\right)+c_{A l} M_{A l}$, $c_{\text {wat }}=4185 \mathrm{~J} /(\mathrm{kg} \cdot \mathrm{K}), \quad c_{\text {Al, solid }}=903 \mathrm{~J} /(\mathrm{kg} \cdot \mathrm{K})$, $c_{\text {Al, liquid }}=1090 \mathrm{~J} /(\mathrm{kg} \cdot \mathrm{K}) \quad c_{\mathrm{H}_{2} \mathrm{O}}=2600 \mathrm{~J} /(\mathrm{kg} \cdot \mathrm{K})$, $c_{\mathrm{H}_{2}}=14300 \mathrm{~J} /(\mathrm{kg} \cdot \mathrm{K}), \quad \rho_{k}=2600 \mathrm{~kg} / \mathrm{m}^{3}$, $R=8.31 \mathrm{~J} /(\mathrm{mol} \cdot \mathrm{K}), \eta=0.00002 \mathrm{~Pa} \bullet, \lambda_{2}=1 \mathrm{Bm} /(\mathrm{m} \cdot \mathrm{K})$, $k_{A l, 1}=1.2 \cdot 10^{-10} \mathrm{~m}^{2} / \mathrm{s}, \quad k_{A l, 2}=1.2 \cdot 10^{-8} \mathrm{~m}^{2} / \mathrm{s}, \quad T_{1,0}=270 \mathrm{~K}$, $Q_{A l}=15.0 \mathrm{MJ} / \mathrm{kg}[5], \Lambda=332400 \mathrm{~J} / \mathrm{kg}, L=1712940 \mathrm{~J} / \mathrm{kg}$, $c_{2}=m_{H_{2} O} c_{H_{2} \mathrm{O}}+m_{H_{2}} c_{H_{2}}, \quad \mu_{A l}=0.027 \mathrm{~kg} / \mathrm{mol}$, $\mu_{O}=0.016 \mathrm{~kg} / \mathrm{mol}, \mu_{\mathrm{H}_{2}}=0.002 \mathrm{~kg} / \mathrm{mol}, P=4 \div 20 \mathrm{MPa}$.

In the mathematical model (1)-(19), the coefficient $k_{A l, i}$ was considered as the only coefficient used for fitting the 
computed results and experimental data. The coefficient was reconciled by comparing the calculated burning rate of a frozen nano-aluminum-water suspension (ALICE fuel) with experimental data [7]. The calculation results of the burning rate for the same suspension depending on pressure are presented in [2].

The present paper is devoted to combustion of a frozen aluminum-water suspension with nano- and micro-dispersed particles at a stoichiometric condition. We conducted the parametric investigation on the linear burning rate depending on the mass ratio of nano- and micro-dispersed powders in the suspension.

\section{Results}

The results of numerical investigation and its comparison to the experimental data obtained by the authors in [1] are shown in Figures 1 and 2. The figures provide the linear burning rate of a frozen Al-water suspension as a function of particle mass fraction ratio. The dots on the plot correspond to the experimental data [1], the line is the result of the numerical investigation. An increase in the mass fraction of a micron-sized powder leads to a decrease in the burning rate. At the same time, the larger the size of the micron particles in the suspension, the higher the sensitivity of the burning rate to the increase in its mass fraction in the composition of the suspension. The results of the numerical simulation using the model (1) - (20) agree with the data presented in the scientific literature [1].

\section{Conclusion}

A mathematical model of solid fuel combustion based on a frozen nano- and micro-dispersed aluminum suspension with water is developed, which makes it possible to estimate the burning rate value within a wide pressure range. The results of the calculations agree satisfactorily with the experimental data published in the scientific literature.

The results to be obtained will be useful for the development of fundamental knowledge in the field of physics of combustion HEMs to calculate basic internal ballistic characteristics of the propulsion systems and reduce the amount of expensive experiments.

This work was supported by the Russian Science Foundation (Grant No. 17-79-20011).

\section{References}

1. T.L. Connell, G.A. Risha, R.A. Yetter, V. Yang, S.F. Son, International Journal of Energetic Materials and Chemical Propulsion, 3, 11, 259-273 (2012)

2. A.Y. Krainov, V.A. Poryazov, Combustion, Explosion and Shock Waves, 52, 2, 177-183 (2016)

3. A.Y. Krainov, V.A. Poryazov, Journal of Engineering Physics and Thermophysics, 89, 3, 579586 (2016)
4. A.F. Belyaev Combustion Theory of Powders and Explosive (Nauka, Moscow, 1982)

5. D.A. Yagodnikov Ignition and Combustion of Powdered Metals (Bauman Moscow State Technical University Publishing, Moscow, 2009)

6. V.G. Prokof'ev, V.K. Smolyakov, Combustion, Explosion and Shock Waves, 38, 2, 143-147 (2002)

7. T.L. Pourpoint, T.D. Wood, M.A. Pfeil, J. Tsohas, S.F. Son, International Journal of Aerospace Engineering. Article ID 874076 (2012) 